\title{
STUDY DESIGN, SIZE, AND ADEQUATE EXPOSURE DATA AS THE CRUCIAL ASPECTS IN CANCER RISK ASSESSMENT AND IMPLEMENTATION OF THE PRECAUTIONARY PRINCIPLE
}

\author{
Vladimír Bencko, Milan Tuček, John M. Quinn \\ Institute of Hygiene and Epidemiology, First Faculty of Medicine, Charles University and General University Hospital in Prague, Prague, \\ Czech Republic
}

\begin{abstract}
SUMMARY
Traditional approaches and study design in cancer epidemiology have not been very successful in identifying and evaluating adequately the potential risk and/or protective factors associated with the disease. The main reasons for the failure are often due the small study sample size, and inadequate exposure information. In this paper, issues and approaches relevant to these two challenges are discussed.

Multicentre study is proposed as a way to increase study size and to mitigate criticism about meta-analysis of independent studies. A multicentre study of large cohort or case-control studies also offer an exciting opportunity to study the contribution of epigenetic events that may be associated with lifestyle and environmental risk factors for human health. Optimizing methods for exposure assessment and how to reduce exposure to misclassification represent a difficult component in epidemiological studies. A potentially useful approach for improving exposure estimation is to rely on biomarkers of exposures. An example is provided to demonstrate how biomarkers of exposures could provide valuable information in addition to exposure measurements in traditional epidemiological studies.

Finally, it is argued that risk assessment and the precautionary principle should not be viewed as conflicting paradigms but, rather, as a complementary approach for developing appropriate policies to address risks posed by exposure to carcinogens and a wide spectrum of other health hazards.
\end{abstract}

Key words: epidemiological data, risk assessment, precautionary principle, cancer epidemiology, carcinogenicity, multicentre studies, genomics of cancer, epigenomics

Address for correspondence: V. Bencko, Institute of Hygiene and Epidemiology, First Faculty of Medicine, Charles University, Studničkova 7 , 12000 Praha 2, Czech Republic. E-mail: vladimir.bencko@lf1.cuni.cz

https://doi.org/10.21101/cejph.a6159

\section{INTRODUCTION}

The epidemiology of neoplasms found itself at the crossroads of new challenges and chances. While the major carcinogenic factors in the work environment have been identified, we are now confronted with a long list of potential, suspicious carcinogens for which epidemiological data are either absent or not yet adequate. The number of commercially used chemicals is estimated between tens of thousands and more than 140,000 items (1). Most of them lack relevant toxicological information to establish the required hygiene standards/maximum allowable concentrations in the air or drinking water, or acceptable daily food rations (2). Carcinogens classified in the IARC/WHO classification were originally identified in the working environment because workers were exposed to concentrations/doses at workplaces by at least one, often more orders of magnitude, higher than the general population. After the adoption of relevant legislation in the field of occupational health, the exposure to classical carcinogens, especially in the workplace, has usually been dramatically reduced.
Advances in the disciplines on which the methods of estimating the health risk of exposure depend are a promising prerequisite for understanding the impact of harmful factors on human health. In recent decades, considerable progress has been made in the area of health risk assessment, as well as in the broad field of cancer epidemiology. This includes not only research into exposure to carcinogens in the living and working environment, but increasing emphasis is also placed on lifestyle risk factors including nutrition. Traditional epidemiological approaches in epidemiology in a wide range of suspected carcinogens have failed due to insufficient coverage of investigated individuals and inadequate data describing their exposure levels.

\section{Increasing the Number of Probands - Multicentre Studies}

Over the past decades, the number of collaborative studies has increased sharply, leading to a substantial increase in the number of probands in epidemiological studies. This led to an increase in the strength of these studies, i.e. the hope to identify significant 
associations between exposure and the clinical parameter studied - in our case one of the malignancies studied. For example, when considering occupational lifelong exposure, it is very likely that in the case of the general population, its share in the population under investigation will be low (most often below 5, but not more than $10 \%)$. For this reason, at least 1,000 cases and the same number of controls must be investigated in order to identify the statistically significant causal relationship between exposure and the malignancy studied $(2,3)$. Such numbers of cases and controls are usually difficult to obtain in individual hospitals or centres during the time spent on research projects, usually 3 , but not more than 5 years, including the necessary "cleanup of databases" and evaluation of the data obtained. Therefore, the most common solution to this problem today is multicentre studies. In addition to a significant increase in strength, studies provide additional benefits, in particular a wider range of exposure to the factors under consideration, which is most useful for analysing the dose (exposure rate) and effect analysis and provides an opportunity to analyse observed exposure differences between participating centres, regions or countries.

Multicentre studies can be conducted in essentially two ways. Collaboration can be initiated after completion of individual studies (retrospectively) or prior to their commencement, which is a methodically more advantageous alternative in the evaluation phase of the results database. Prospective studies have become possible since international institutions, in our case the European Commission, have started to offer the possibility of supporting these costly projects. A key advantage of this type of study is the unified data collection protocol in all participating centres, which virtually eliminates the unwanted lack of important information in the data pooling phase prior to their statistical analysis. This, on the other hand, is a serious problem in retrospectively performed meta-analyses.

From the perspective of Central and Eastern European countries, an important example of these studies is the multicentre study on lung cancer, organized by IARC/WHO Lyon, France, and the follow-up study under an identical protocol on renal malignancies also supported by NCI, Bethesda, USA $(3,4)$. The first of the two studies was supported by the 4th EC Framework Programme and involved 3 centres from the Czech Republic, 2 centres from Poland, and one centre from Hungary, Romania, the Russian Federation, and Slovakia.

\section{Refinement of Exposure Rate Estimation}

Optimization of exposure estimation methods and reduction of their undesirable deviations from reality, misclassification, is the most difficult component of epidemiological studies of this type. A perfect estimation of exposure rates for long-latency diseases, to which malignant neoplasms undoubtedly belong, would be their quantitative measurement over the entire exposure period, an unrealistic utopia in the foreseeable future. The availability of group exposure data at several time intervals is assessed as an exceptional, "luxury" situation for professional exposure in relatively rare cases, in epidemiological studies based on the general population.

In population and case studies, exposure to carcinogenic factors can only be estimated retrospectively. As a result, these estimates are based on information concerning the profession of probands obtained from them or their close relatives. The information can be specified individually, on a case-by-case basis, by an experienced occupational hygiene or occupational health expert, familiar with the situation in the regional industry. However, the subjective nature of the estimation and the limited use of objectively measured data on exposure rates in these studies are their main weakness, as there is a real risk of misrepresentation of probands' exposure rates in the absence of a "gold standard" (5-8).

A significant chance of improving this difficult situation is to abandon the raw smoker/non-smoker exposure parameters, generally exposed/unexposed, with a more precise quantified estimate covering exposure time (e.g. a parameter that has been a traditional estimate of cigarette packs for many years). Data of this kind are then valuable in evaluating the dose/effect relationship, which is of key importance in deciding the possible causality of the relationship. These structured data make it possible to compare the results of data analysis collected by individual centres, taking into account the type of industry, profession, or lifestyle and nutrition risk factors under standardized conditions.

In this context, biomarkers of exposure, which have been used for decades but have not yet been fully exploited, are of growing importance. This potential is evident when the studied factor can enter the human body in several ways (e.g. toxic metals), or sources of exposure are difficult to evaluate (e.g. passive smoking). There are situations where biomarkers of exposure provide valuable information even in classical epidemiological studies, e.g. when studying exposure of local populations exposed to arsenic in drinking water. Its concentration in water is determined, but the estimation of exposure is dependent on a number of parameters - namely the amount of water consumed and the water used in cooking, from which the daily intake is estimated. A useful objective solution to this problem is to determine arsenic in hair, nails, urine, or blood. The suitability of each variant must be considered in terms of chronic or acute exposure levels. Health risk cannot be assessed from the "sui generis" parameters, but they illustrate their potential usefulness in a realistic estimate of the exposure rate and dispersion of the individuals in the sample.

\section{Epigenomics, New Chance and Opportunities}

While the genetics of malignant neoplasms has been studied in recent decades and a considerable number of epidemiological studies have been devoted to it so far, the evaluation and practical application of epigenetic aspects of these studies is a relatively recent matter. The study of the role of epigenetic changes induced by xenobiotics from the environment, food and lifestyle risk factors is still at an early stage. To date, little is known about the mechanism for human health of adverse epigenetic disorders induced by the environment or diet. While there is ample evidence that aberrant DNA methylation may be due to exposure to epimutagens, we still have little evidence of the consequences of stimulation caused by hereditary changes stored in histones. Epigenetics is still little explored, but all the more attractive field for serious research. Although it seems inevitable that some histone disorders or modifications may be induced by diet or environmental factors, and perhaps contribute to the development of chronic diseases, including malignant neoplasms, reliable evidence of the feasibility of such suspicions has been lacking. Unlike classical genetic 
changes, epigenetic alterations are reversible and progressive. This is the main reason for their intensive study and their potential for use in primary prevention strategies (9).

Multicentre and extensive cohort studies offer promising opportunities to study the effects of dietary epigenetic factors and cancer-inducing environments. An example of such an advanced stage study is the European Prospective Study on Nutrition and Cancer, supported by the European Commission, conducted in 10 European countries (10), and the above-mentioned lung and kidney cancer studies in Central and Eastern Europe and similar studies of nasopharyngeal malignancies $(11,12)$. These studies include several thousand volunteers and represent a unique chance to identify risk or protective environmental factors, diet and lifestyle.

Among the protective ones, the use of Mendelian randomization was able to prove the protective effect of cross-border plants in diet in case of lung and kidney cancer, together with an explanation of the mechanism of their projective action. This is inversely dependent on the expression of glutathione S-transferase genes $(3,4)$.

\section{Estimating Risk Rate}

Risk estimation is a rapidly evolving field and its methods of calculation are undergoing remarkable development. The delay between exposure and effect, together with the late effects of exposure (cessation lag and lingering effect) are important aspects of the current approach to addressing the assessed situations and are relevant for assessing the dose/effect relationship in the context $(13,14)$. Moreover, by taking into account the biological mechanism of action of the relevant factor, these concepts are useful in analysing the economic benefits of a possible subsequent intervention. The concept of cumulative and late effects can be used in the analysis of epidemiological data to reveal hidden biological connotations with the neoplasm under investigation, thus supporting current efforts to characterize risk and ultimately reduce uncertainties in estimating the degree of risk.

Controversies literally teeming with methodological approaches used to estimate the level of health risks include, in particular, extrapolations beyond the range of available exposure data, particularly below the usual "threshold dose" area, underlined by uncertainties and limitations in the use of observational studies $(15,16)$ and implications of these estimates (2). Sceptics argue that risk assessment, as it stands today, is not an adequate tool for estimating health risk from exposure to risk factors in the environment and the work environment (17). Their main objection is that intensifying debates around this problem may be abused and lead to undesirable delays in the relevant legislative measures.

As a deterrent from legislative practice, an estimate of the risk of carcinogenicity of particulate matter in diesel engine emissions, which has remained at the US Environmental Protection Agency for 20 years due to various objections and at least in part sceptical doubts from experts in various professions. It cannot be ruled out that probably a considerable part of them worked for the benefit of the manufacturers of these engines. Until a technical solution to the problem leading to emission reductions has been found, the adoption of the relevant emission limits has been blocked by an endless series of objections (18).

Excited debates arose a few years before the adoption of the "precautionary principle", which was adopted as an alternative basis for European environmental and health legislation and also for consumer protection $(19,20)$. This principle is defined as the need to take precautionary measures to avert human health threats, even if the harmful effect of this factor is not reliably, definitively established at the time, according to the principles of "evidence-based medicine". From an epidemiological point of view, this is not a "novelty".

John Snow persuaded the local authority to close the Broad Street pump to stop the cholera epidemic around it for years before Robert Koch discovered Vibrio cholerae and proved that it was indeed a waterborne infection. This already classic story highlights the importance of taking preventive measures at a time when we are faced with a number of uncertainties, after considering the risk of not taking them. We do not regard both the "Health Risk Assessment" and the "Precautionary Principle" as necessarily conflicting paradigms, but they can be seen as complementary "ad hoc" approaches based on the amount of information available when it is needed, often in an emergency, decide to take preventive measures. Usually exposure to carcinogens is not an emergency like epidemics or natural or industrial disasters or accidents, but our unenviable position in the incidence of renal and colorectal cancer malignancies and some of the less frequent malignancies in our population should be a rational introduction to intensifying not only research work, but also encouraging efforts in the field of primary prevention in this field. The gaping scissors between the growing incidence and the indicated stagnation in mortality from these malignancies is an undisputed success of early secondary prevention, i.e. early diagnosis and successful therapy, as opposed to lame, underfunded primary prevention.

\section{CONCLUSION}

Epidemiological data play a key role in the classification of human carcinogens. The intensive involvement of genomic aspects, as an integral and irreplaceable item in epidemiological studies of chronic diseases, together with the adoption of the current concept of multicentre studies and large-scale cohort studies, reinforces the hope that clinically useful biomarkers will be identified as well as new strategies not only for health risk analysis but also for the primary prevention of these diseases including malignancies $(21,22)$. Prevention, in particular primary prevention of diseases, is one of the declared priorities of public health in the 21 st century.

\section{Acknowledgements}

This paper was elaborated within activities supported by research intention PROGRES Q29/LF1.

\section{Conflict of Interests}

None declared

\section{REFERENCES}

1. Bencko V, Chen C. Epidemiology data and cancer risk assessment: cessation lag and lingering effect concepts. Cent Eur J Public Health. 2014;22(3):197-200.

2. Stayner L. Boffetta P. Vainio H. Risk assessment of carcinogenic hazards In: Schottenfeld D, Fraumeni JF Jr, editors. Cancer epidemiology and prevention. 3rd ed. Oxford: Oxford University Press; 2006. p. 65-9. 
3. Brennan P, Hsu CC, Moullan N, Szeszenia-Dabrowska N, Lissowska J, Zaridze D, et al. Effect of cruciferous vegetables on lung cancer in patients stratified by genetic status: a mendelian randomisation approach. Lancet. 2005 Oct 29-Nov 4;366(9496):1558-60.

4. Moore LE, Brennan P, Karami S, Hung RJ, Hsu C, Boffetta P, et al. Glutathione S-transferase polymorphisms, cruciferous vegetable intake and cancer risk in the Central and Eastern European Kidney Cancer Study. Carcinogenesis. 2007 Sep;28(9):1960-4.

5. Thompson SG, Pocock SJ. Can meta-analyses be trusted? Lancet. 1991 Nov 2;338(8775):1127-30.

6. Blettner M, Sauerbrei W, Schlehofer B, Scheuchenpflug T, Friedenreich C. Traditional reviews, meta-analyses and pooled analyses in epidemiology. Int J Epidemiol. 1999 Feb;28(1):1-9.

7. Stroup DF, Berlin JA, Morton SC, Olkin I, Williamson GD, Rennie D, et al. Meta-analysis of observational studies in epidemiology: a proposal for reporting. Meta-analysis Of Observational Studies in Epidemiology (MOOSE) group. JAMA. 2000 Apr 19;283(15):2008-12.

8. Benke G, Sim M, Forbes A, Salzberg M. Retrospective assessment of occupational exposure to chemicals in community-based studies: validity and repeatability of industrial hygiene panel ratings. Int J Epidemiol. 1997 Jun;26(3):635-42.

9. Herceg Z. Epigenetics and cancer: towards an evaluation of the impact of environmental and dietary factors. Mutagenesis. 2007 Mar;22(2):91-103.

10. Riboli E, Kaaks R. The EPIC Project: rationale and study design. European Prospective Investigation into Cancer and Nutrition. Int J Epidemiol. 1997;26 Suppl 1:S6-14

11. Hung RJ, van der Hel O, Tavtigian SV, Brennan P, Boffetta P, Hashibe M. Perspectives on the molecular epidemiology of aerodigestive tract cancers. Mutat Res. 2005 Dec 30;592(1-2):102-18.

12. Scélo G, Constantinescu V, Csiki I, Zaridze D, Szeszenia-Dabrowska N, Rudnai P, et al. Occupational exposure to vinyl chloride, acrylonitrile and styrene and lung cancer risk (Europe). Cancer Causes Control. 2004 Jun; 15(5):445-52.

13. Chen CW, Gibb H. Procedures for calculating cessation lag. Regul Toxicol Pharmacol. 2003 Oct;38(2):157-65.

14. Chen $\mathrm{CW}$. Lingering effect: epidemiological information useful for risk assessment. Regul Toxicol Pharmacol. 2008 Dec;52(3):242-7.

15. Bencko V. Risk assessment and human exposure to endocrine disrupters. In: Jedrychowski WA, Perera FP, Maugeri U, editors. Molecular epidemiology in preventive medicine. Luxemburg: International Center for Studies and Research in Biomedicine; 2003. p. 315-27.

16. Bencko V, Rames J, Ondrusova M, Plesko I, Jurickova L, Trnovec T. Human exposure to polyhalogenated hydrocarbons and incidence of selected malignancies - central European experience. Neoplasma. 2009;56(4):353-6.

17. Silbergeld EK. Risk assessment: the perspective and experience of U.S. environmentalists. Environ Health Perspect. 1993 Jun;101(2):100-4.

18. Stayner L. Protecting public health in the face of uncertain risks: the example of diesel exhaust. Am J Public Health. 1999 Jul;89(7):991-3.

19. Commission of the European Communities. Communication from the Commission on the precautionary principle. Brussels: Commission of the European Communities; 2000.

20. Kriebel D, Tickner J. Reenergizing public health through precaution. Am J Public Health. 2001 Sep;91(9):1351-5.

21. Bencko V. Use of epidemiological data for cancer risk assessment: approaches, concepts, and issuess. Open Epidemiol J. 2011 Jan 19;4:94-8. doi: 10.2174/1874297101104010094.

22. Bencko V. Health risks assessment and a psycho-somatic and psychosocial aspects of risk perception. Hygiena. 2017:62(3):90-3. (In Czech.)

Received February 29, 2020 Accepted in revised form August 28, 2020 